\title{
Spin physics highlights from STAR
}

\author{
A. Gibson ${ }^{1, a}$, on behalf of the STAR Collaboration \\ ${ }^{1}$ Valparaiso University, Valparaiso, Indiana, 46383, USA
}

\begin{abstract}
As the world's only polarized proton collider, the Relativistic Heavy Ion Collider (RHIC) at Brookhaven plays an important role in understanding the spin structure of the proton. The STAR detector, with its large acceptance for calorimetry and tracking, has been used to study polarized proton collisions for more than a decade with a range of jet, meson, and boson probes. We will discuss jets, neutral pions, and $\mathrm{W}$ bosons as probes of the proton's helicity structure. Here STAR measurements have significant impact on global fits of sea quark polarizations and have provided the first firm evidence of non-zero gluon polarization within the proton. We will discuss $\mathrm{W} / \mathrm{Z}$ bosons, jets, pions, and pion-jet correlations as probes of the transverse spin structure of the proton, and we will use the example of a proposed dijet measurement with an upgraded STAR detector to peer into the future.
\end{abstract}

\section{Introduction}

Understanding the proton's spin structure is a fundamental goal of QCD and of nuclear physics. When polarized deep-inelastic scattering experiments in the 1980's [1] showed that only about $30 \%$ of the proton's spin was carried by quarks, as compared to naive expectations from the proton's linear momentum distribution, or from the charge distribution where quarks are the entire effect, the result was dubbed the proton spin "crisis". Or, more prosaically, the proton spin puzzle. Equally puzzling, in the 1970's large transverse spin asymmetries were observed in forward pion production from polarized proton collisions in a fixed target experiment [2], when, naively, extremely small transverse asymmetries were expected. The decomposition of the proton's spin, particularly the possible contributions from gluons and orbital angular momentum, and the mechanisms responsible for the large forward transverse spin asymmetries have continued to be active topics of experimental and theoretical investigation. A recent review of spin physics provides further background [3].

As the world's only polarized proton collider, the Relativistic Heavy Ion Collider (RHIC) at Brookhaven National Laboratory has an important role to play in understanding the spin structure of the proton. The STAR detector [4], with excellent tracking coverage from its time projection chamber (TPC) in the pseudorapidity region $|\eta|<1.3$, extensive electromagnetic calorimeter coverage with $-1<\eta<4$ and $2 \pi$ azimuthal acceptance is well-suited to take advantage of the collisions provided by RHIC. The large-acceptance tracking and calorimetry coverage combine to make STAR an excellent detector for jets, the EM calorimeter coverage allows reconstruction of neutral mesons to forward $\eta$, and the RHIC energy of up to $\sqrt{s}=510 \mathrm{GeV}$ allows the production of $W$ bosons. We will discuss

\footnotetext{
${ }^{\mathrm{a}}$ e-mail: adam.gibson-even@ valpo.edu
} 
jet and pion probes of the gluon polarization within the proton in Section 2, studies of the sea quark polarization using W bosons in Section 3, probes of the proton's transverse structure in Section 4, and we will use the example of a proposed dijet measurement with an upgraded STAR detector to peer into the future in Section 5.

An overview of the heavy ion physics program at STAR can be found in these same proceedings [5].

\section{Probing the Gluon Polarization with Jets and $\pi^{0}$ 's}

The gluon polarization within the proton can be accessed in polarized proton collisions with strongly interacting probes such as inclusive jet or hadron production by measuring the longitudinal doublespin asymmetry $\left(A_{L L}\right)$,

$$
A_{L L}=\frac{\sum\left(P_{B} P_{Y}\right)\left(N^{++}-r N^{+-}\right)}{\sum\left(P_{B} P_{Y}\right)^{2}\left(N^{++}+r N^{+-}\right)},
$$

in which $P_{B, Y}$ are the measured beam polarizations, $N^{++}$and $N^{+-}$are the inclusive jet (or hadron) yields for equal and opposite proton beam helicity configurations, and $r$ is the relative luminosity. Each sum is over individual data acquisition runs that were 10 to 60 minutes long, a period much shorter than typical time variations in quantities such as $P_{B, Y}$ and $r$. Values of $r$ were measured runby-run, and range from 0.8 to 1.2 . A typical beam polarization is about $50 \%$.

Early asymmetry measurements from STAR and PHENIX were included in the DSSV [6] global polarized parton distribution function (PDF) fit, the first to include new RHIC data along with data from fixed-target experiments. For the 2009 run, improvements at STAR, including larger jet trigger efficiency, and additional luminosity allowed a nearly 20 -fold increase in the sample size for the inclusive jet measurement and a corresponding reduction in the uncertainty on $A_{L L}$. Jets are reconstructed utilizing the anti- $k_{T}$ jet-reconstruction algorithm [7] with a radius of 0.6 and utilize energy deposition in the barrel (BEMC) and endcap (EEMC) electromagnetic calorimeters as well as charged-particle tracks from STAR's TPC. This result is presented in Fig. 1 and has recently been submitted for publication [8]. The updated result has been included in a new fit by the DSSV collaboration [9] as also shown in Fig. 1. The integral over gluon momentum fraction $0.05<x<1$ yields a result of $0.194_{-0.054}^{+0.057}$ [10], the first firm evidence of a non-zero role for the gluon in determining the spin of the proton. By contrast, the role of the gluon at low $x$ remains quite poorly constrained.

Using the 2006 dataset, STAR also measured $A_{L L}$ for neutral pions, with their different kinematics, fragmentation, and systematics at central $(|\eta|<0.95)$ [11] and forward $(\eta=3.2,3.7)$ [12] pseudorapidity. In a recent publication [13] we extended those measurements to the EEMC at intermediate $\eta, 1.0<\eta<2.0$, still using the 2006 dataset at $\sqrt{s}=200 \mathrm{GeV}$. These results are presented in Fig. 2. Another recent global QCD analysis, by the NNPDF collaboration [14] incorporates RHIC jet measurements, and makes predictions for $\pi^{0}$ observables. In Fig. 3 we show their prediction for our EEMC measurement. Clearly improvements in statistics are needed to further constrain the global fit. To extend STAR's sensitivity, including extending our $\Delta g(x)$ reach to lower $x$ values, we have several options. We can increase the statistics, we can increase the beam energy, we can probe lower $p_{T} \pi^{0}$ 's (limited by our ability to trigger and reconstruct these events), and we can push to forward $\eta$. We plan to pursue several of these strategies with $\pi^{0}$ 's in the EEMC and forward meson spectrometer (FMS) [15], where a lack of tracking coverage makes jet measurements difficult. Projected statistical uncertainties for a measurement with EEMC $\pi^{0}$ 's from the 2012 dataset are shown in Fig. 3. This 2012 dataset is at $\sqrt{s}=510 \mathrm{GeV}$ with a factor of 10 larger luminosity than the 2006 result. 

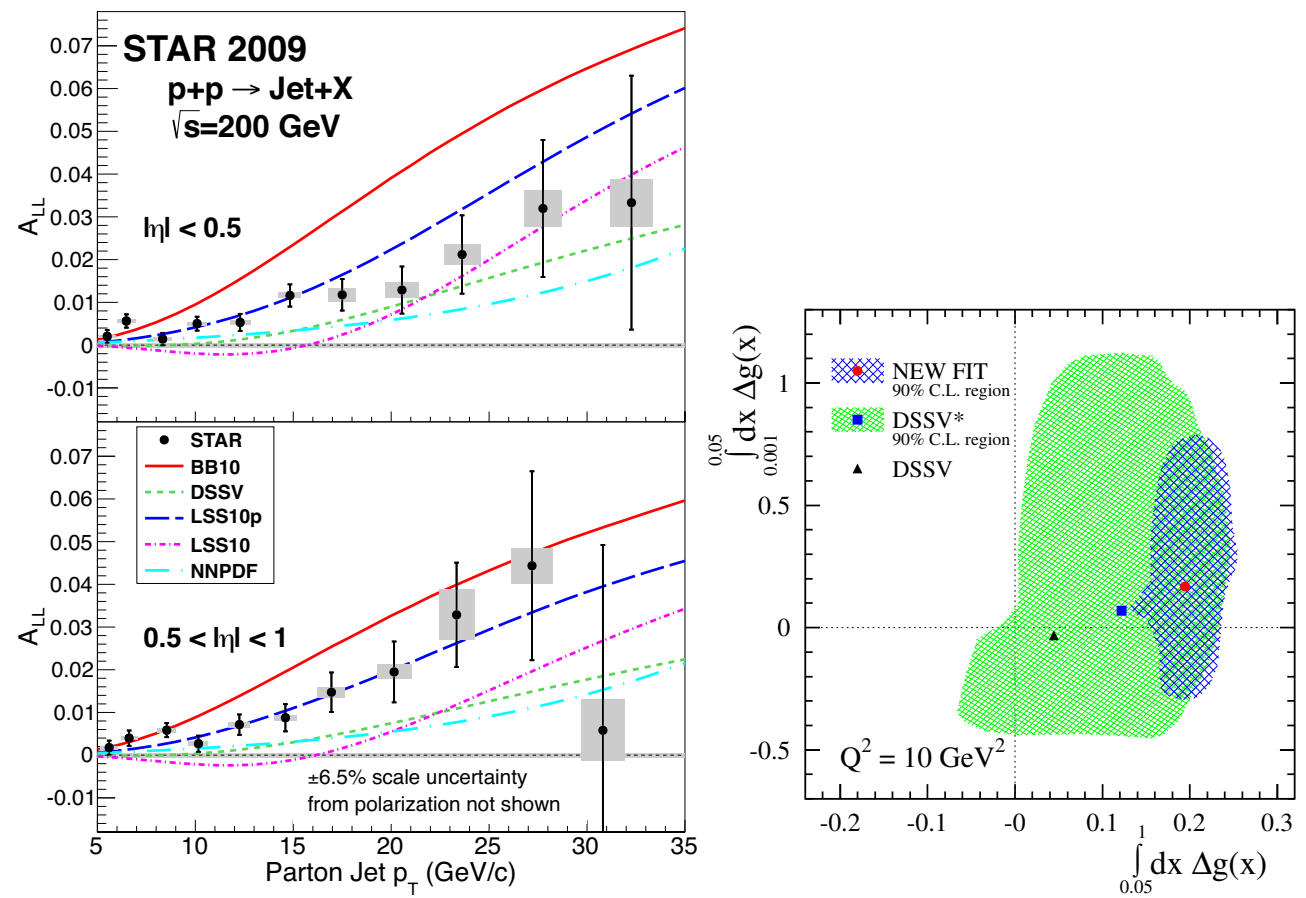

Figure 1. (Left) Midrapidity $(|\eta|<0.5$, upper panel) and forward rapidity $(0.5<|\eta|<1$, lower panel) inclusive jet $A_{L L}$ vs. parton jet $p_{T}$, compared to predictions from several NLO global analyses. The error bars are statistical. The gray boxes show the size of the systematic uncertainties. (Right) The new DSSV fit [9], heavily influenced by these jet results. For high gluon $x$ the fit shows the first firm evidence of non-zero gluon polarization in the proton, while the low- $x$ gluons remain poorly constrained.

\section{Probing Sea Quark Polarizations with W's}

The flavor-separated helicity-dependent PDF's of quarks in the proton can be probed at STAR through the production of $W^{+(-)}$bosons for which the dominant production process is $u+\bar{d}(d+\bar{u})$. The parityviolating weak production gives rise to large single-spin asymmetries, $A_{L}=\frac{\sigma_{+}-\sigma_{-}}{\sigma_{+}+\sigma_{-}}$, where $\sigma_{+(-)}$is the cross section with the helicity positive (negative) proton beam (averaged over the other beam's helicity states). The $W$ bosons are identified by an isolated high- $p_{T}$ electron in the BEMC or EMC in an event with imbalanced $p_{T}$, as expected from the missing neutrino. The electron sign identification is handled by the TPC $(|\eta|<1.3)$. The STAR $W$ results from 2012 have recently been published [17] and are shown in Fig. 4 along with a variety of theoretical predictions. The 2012 dataset is about six times larger than earlier datasets. As seen in Fig. 4, the STAR result has a significant effect on global fits to the sea quark polarizations.

\section{Probing Transverse Structure with Jets, $\pi^{0}$ 's, and Other Probes}

The large transverse asymmetries originally seen in forward charged pion production at low $\sqrt{s}$ [2] have since been observed over a broad range of $\sqrt{s}$, including at RHIC energies and also with $\pi^{0}$ 's, as 


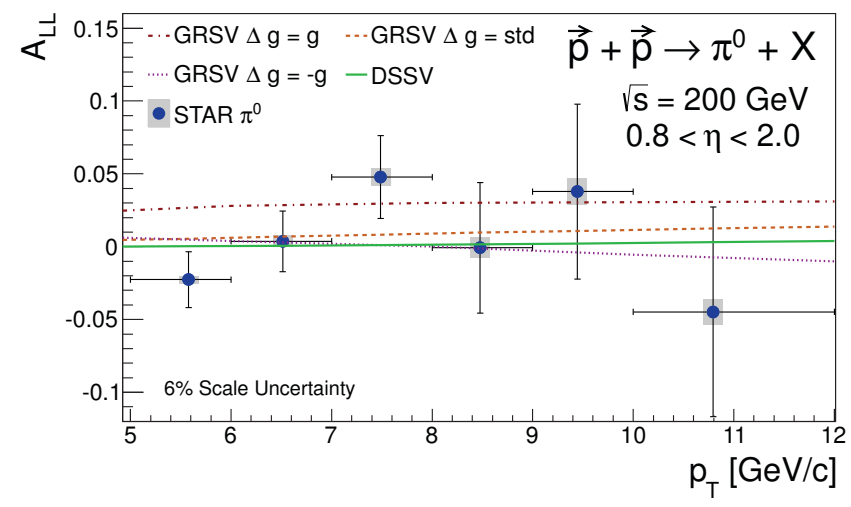

Figure 2. The EEMC $A_{L L}$ results from 2006 data (blue markers) are presented with the DSSV prediction [6] and the GRSV prediction [16] using the best fit to polarized DIS ( $\Delta g=$ std) and the maximum and minimum allowed values for gluon polarization. The GRSV prediction includes no RHIC data. Statistical uncertainties are shown by the error bars, whereas systematic uncertainties are indicated by the error boxes. The $6 \%$ scale uncertainty is due to beam polarization uncertainty.
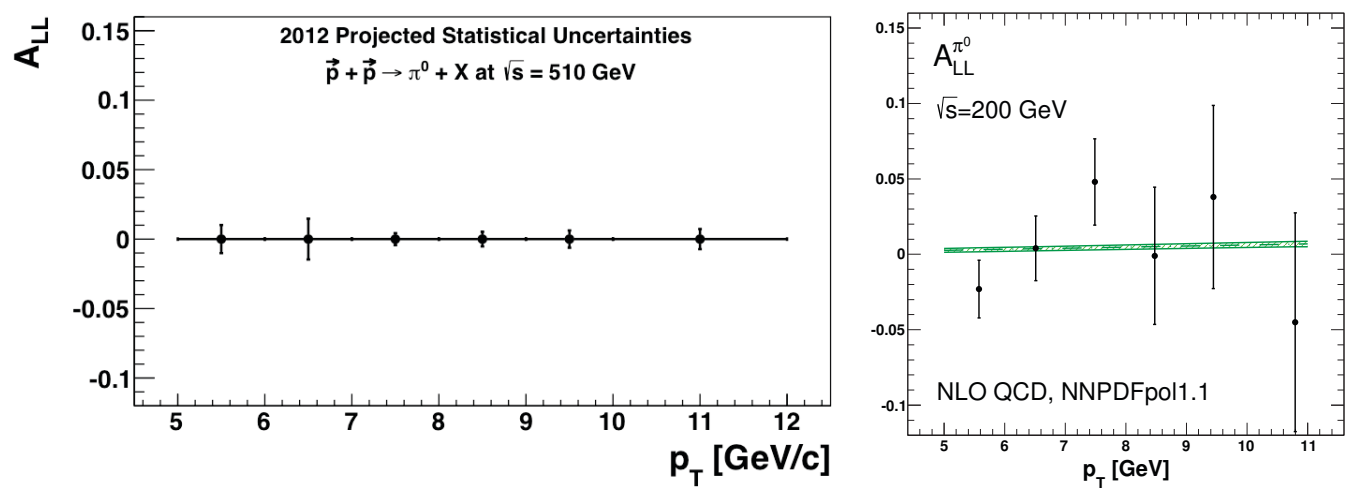

Figure 3. (Left) Projected statistical uncertainties for an inclusive $\pi^{0} A_{L L}$ measurement using the 2012 EEMC dataset, plotted on the same scale as the 2006 result [13] of Fig. 2. (Right) The data points are the 2006 EEMC result, while the green band represent the new NNPDF fit [14], with uncertainty, incorporating recent RHIC jet results.

summarized in Fig. 5. At STAR a number of features of these large asymmetries have been observed including their observation for forward observables but not for central ones, and for relatively isolated $\pi^{0}$ 's but not for jets reconstructed from electromagnetic clusters, their diminution when significant central activity is present in the event, some sign that the asymmetries are larger for $\eta$ 's than for $\pi^{0}$ 's, and, as shown in Fig. 5, their originally unexpected persistence to significant pion $p_{T}$.

To explain the large observed asymmetries in pion production, it is necessary to move beyond the collinear, leading twist, formulation of perturbative QCD [19]. Two approaches have been proposed: 

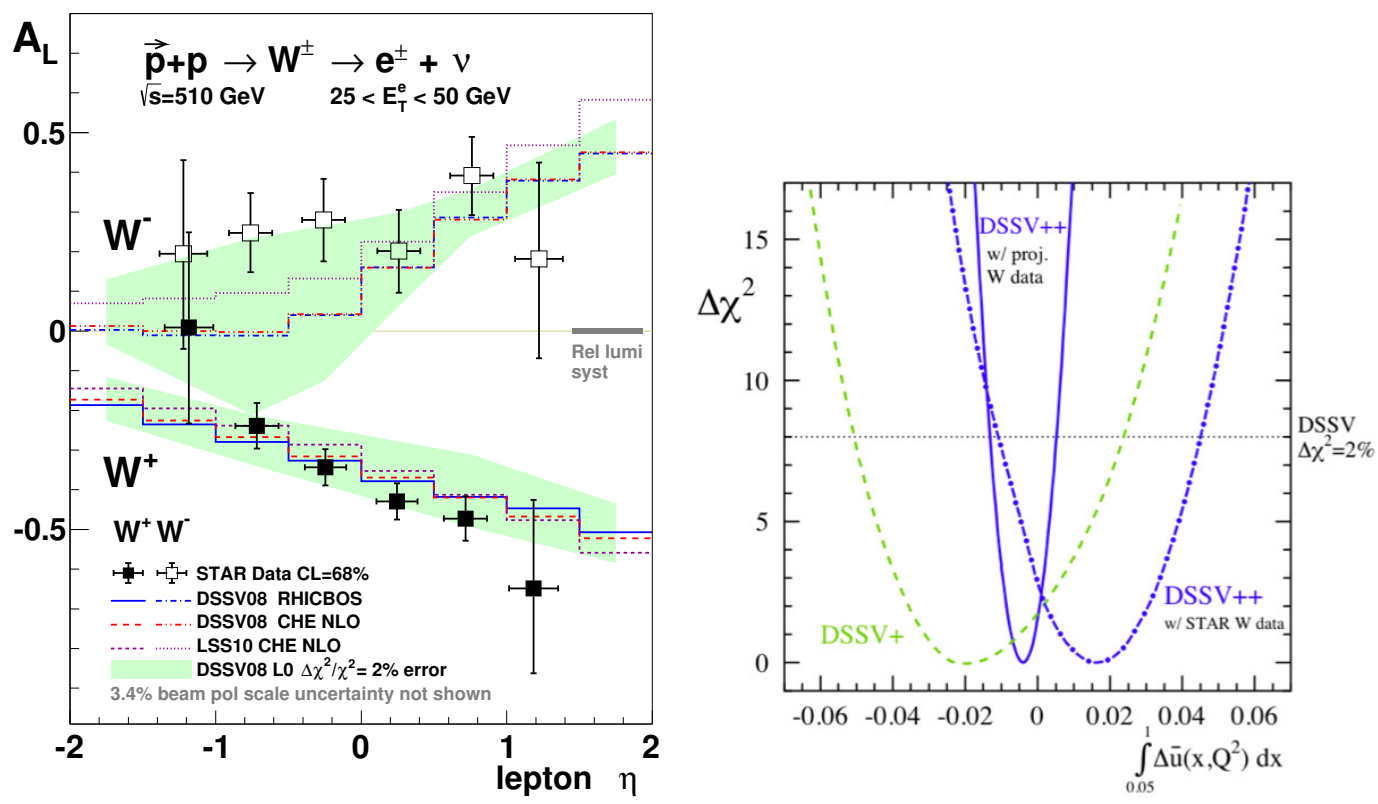

Figure 4. (Left) Longitudinal single-spin asymmetry $A_{L}$ for $W^{ \pm}$production as a function of lepton pseudorapidity, $\eta$, in comparison to theory predictions. (Right) $\chi^{2}$ profiles for $\Delta \bar{u}$ without (DSSV+) STAR W data, with a preliminary version of the 2012 data (DSSV++), and with the preliminary 2012 data along with the uncertainties from projected 2013 results (DSSV++ w/ proj. W data) [18].
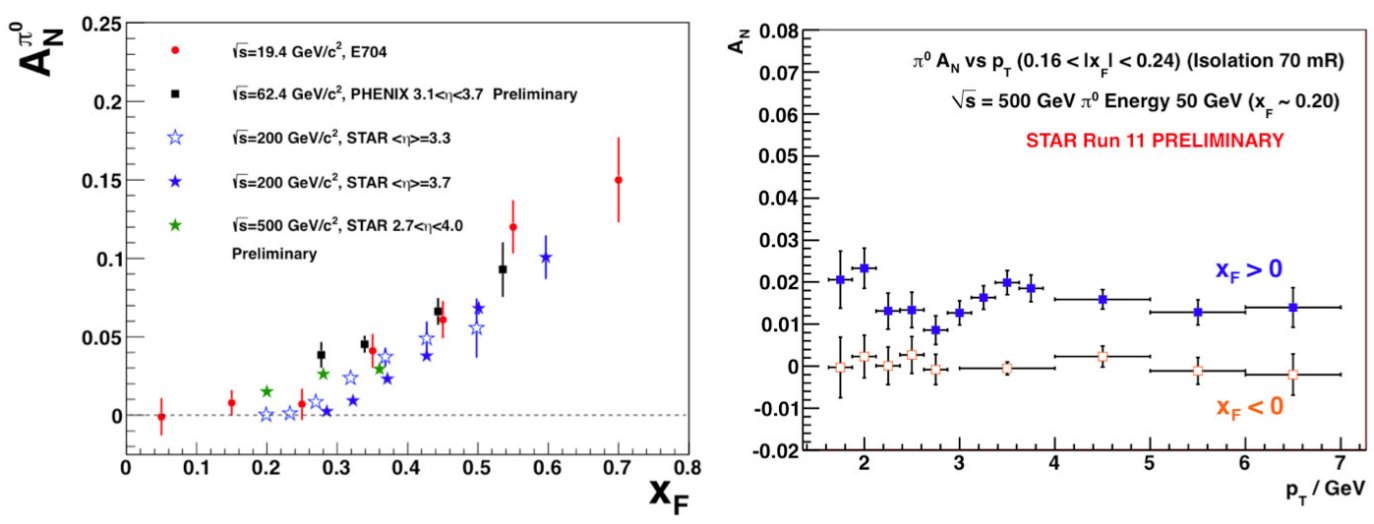

Figure 5. Transverse single spin asymmetry measurements for neutral pions at different center-of-mass energies as a function of Feynman- $x$ (Left) and $p_{T}$ at $\sqrt{s}=500 \mathrm{GeV}$ (Right) [18]. 


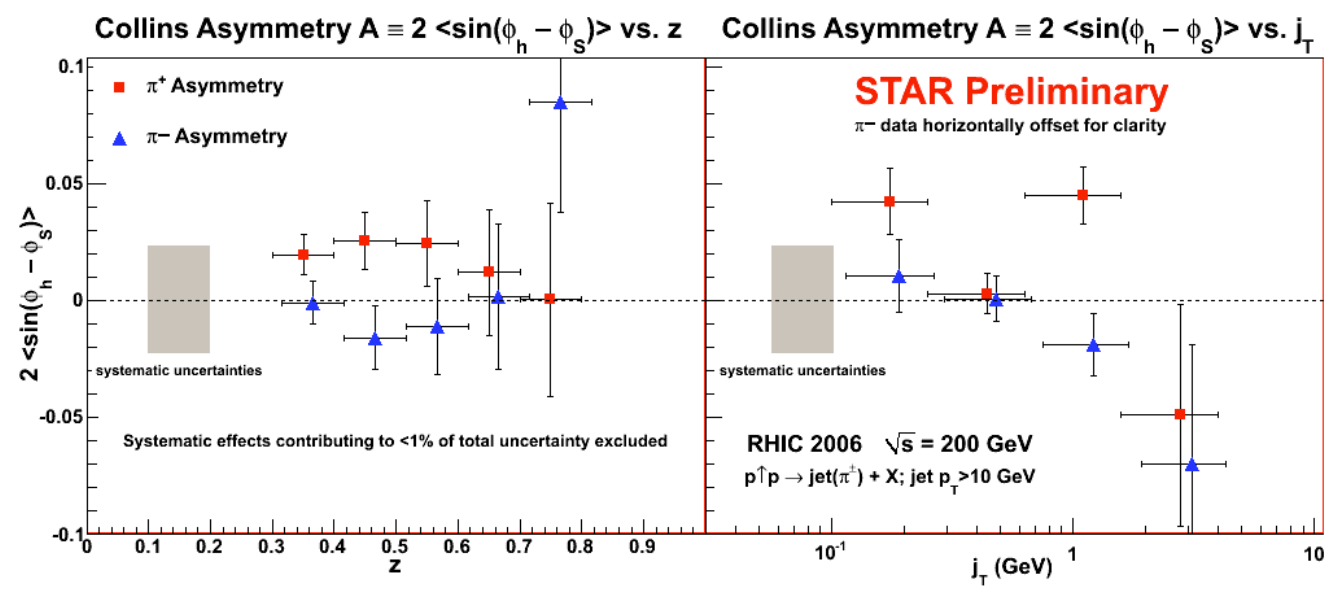

Figure 6. Preliminary results of the Collins moment for leading $\pi^{+}\left(\right.$red) and $\pi^{-}$(blue) particles within midrapidity jets reconstructed by the STAR detector. Statistical errors are shown on data points and the grey shaded band indicates the systematic error bar for the $\pi^{+}$and $\pi^{-}$separately. The momentum fraction of the jet carried by the pion is $z=p_{\pi} / p_{\text {jet }}$.

to account for higher twist multi-parton correlators ("twist-3" formalism) [20, 21] or to account for intrinsic transverse-momentum dependence ("TMD" formalism) [22, 23]. In the twist-3 formalism one can obtain asymmetries, in principle, from either the parton correlation functions or the fragmentation functions (e.g. Refs. [24, 25]). Similarly, in the TMD formalism one can obtain asymmetries, in principle, from either the parton distribution functions (the "Sivers effect" [22, 23]) or the fragmentation functions (e.g. the "Collins effect" [26]). The Sivers effect requires the presence of orbital angular momentum in the proton, while the Collins effect is sensitive to transversity, a fundamental distribution function of the proton. Furthermore, it has been shown that the intrinsic transverse momentum integrals of the TMD functions are closely related to the twist-3 functions [27].

Measurements such as those in Fig. 5 of the transverse asymmetry in inclusive hadrons, or in jets while ignoring the jet substructure, can be sensitive to initial-state effects (such as the Sivers effect). To probe for final-state effects (such as the Collins effect in fragmentation) one can analyze the correlation between the pions fragmenting from a jet, and the jet axis. Such an analysis [28], from a limited statistics $\sqrt{s}=200 \mathrm{GeV}$ dataset in 2006, is shown in Fig. 6. The Collins asymmetry is plotted against $z$ and $j_{T}$, where $z$ is the fraction of the total momentum of the jet carried by the pion and $j_{T}$ is the pion's transverse momentum with respect to the jet axis. These preliminary results show a tantalizing charge-sign separation between the pions, and a hint of a non-zero asymmetry. But, the systematic uncertainties prevent the result from being significant.

At the time of this conference, an analysis with a much larger dataset at $\sqrt{s}=200 \mathrm{GeV}$ from 2012 was underway [29], and a projected statistical uncertainty of its Collins sensitivity is shown in Fig. 7. Another analysis, of a significant dataset at $\sqrt{s}=500 \mathrm{GeV}$ of 2011 data has released preliminary results [30] including the first ever experimental constraints on the "Collins-like" effect from linearly polarized gluons [31]. The preliminary results of the 2011 analysis are consistent with no asymmetry, and also consistent with the preliminary 2006 results, and with theoretical expectations. The 2011 analysis has had its acceptance somewhat enlarged and is proceeding to publication. The preliminary 


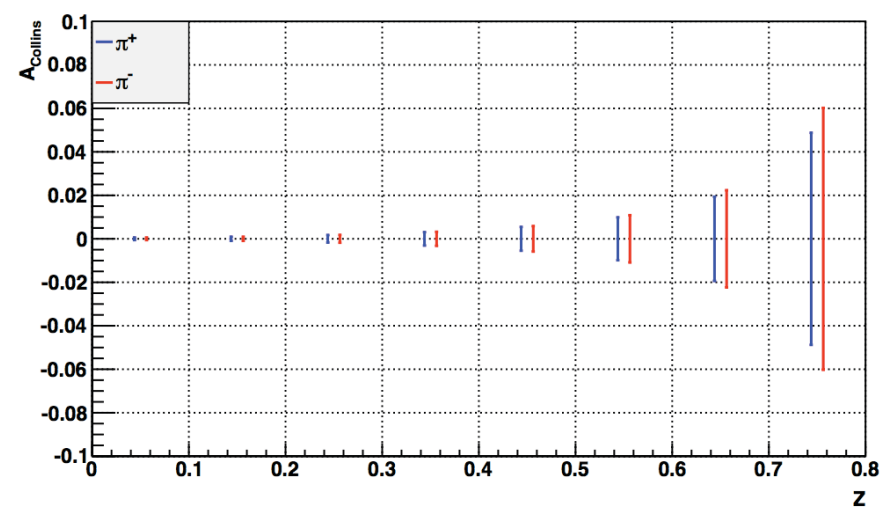

Figure 7. Projected statistical uncertainty of the Collins moment for leading $\pi^{+}$(red) and $\pi^{-}$(blue) particles within mid-rapidity jets reconstructed by the STAR detector, for a significantly larger dataset at $\sqrt{s}=200 \mathrm{GeV}$, like Fig. 6, but from 2012.

results of the 2012 Collins analysis were released after the date of the conference talk described in these proceedings. These preliminary 2012 results are exciting - the first statistically significant Collins measurement at a polarized proton collider - and are described elsewhere [32].

To test the full range of transverse physics phenomenology it is important to test the universality, factorization, and evolution of the various phenomena and formalisms. So, it's important to conduct tests with a range of probes, including changing the initial states (e.g. polarized DIS and semi-inclusive DIS, i.e. SIDIS vs. polarized proton collisions) and probing a range of final states at different $Q^{2}$ scales. One prediction of QCD in the TMD framework is that the Sivers function is not universal, experiencing a sign change as expressed for SIDIS as compared to Drell-Yan [33]. At STAR, it is possible to test this predicted non-universality using heavy boson production, as well as with Drell-Yan itself or direct photon production. For the case of $W$ and $Z$ bosons a preliminary, proof of principle, measurement has been conducted using fully reconstructed $W$ and $Z$ bosons from transversely polarized data recorded in 2011. It is described in more detail elsewhere [34].

\section{Looking to the Future}

We have discussed a rich range of spin physics measurements from STAR at RHIC probing a variety of phenomena using a range of observables and both longitudinally and transversely polarized proton beams. Nevertheless, it does represent a biased selection of highlights, with important STAR results, like the measurement of interference fragmentation functions as evidence for transversity in the proton [35], unfortunately left out. Most of the analyses we have discussed continue, with new preliminary and published results in preparation with datasets from 2011, 2012, and 2013: in many cases promising significantly greater sensitivity. Additional polarized data taking is foreseen in 2015 and 2016, with tests of TMD evolution and universality among the major targets, including the $W$ and $Z$ boson transverse asymmetry results discussed above. An upgrade to the FMS subdetector is currently underway, adding a preshower detector that will allow separation amongst forward $(2.5<\eta<4.0)$ photons, $\pi^{0}$ 's, charged hadrons, and electrons and will support direct photon and Drell-Yan measurements to complement the $W$ and $Z$ measurements. 


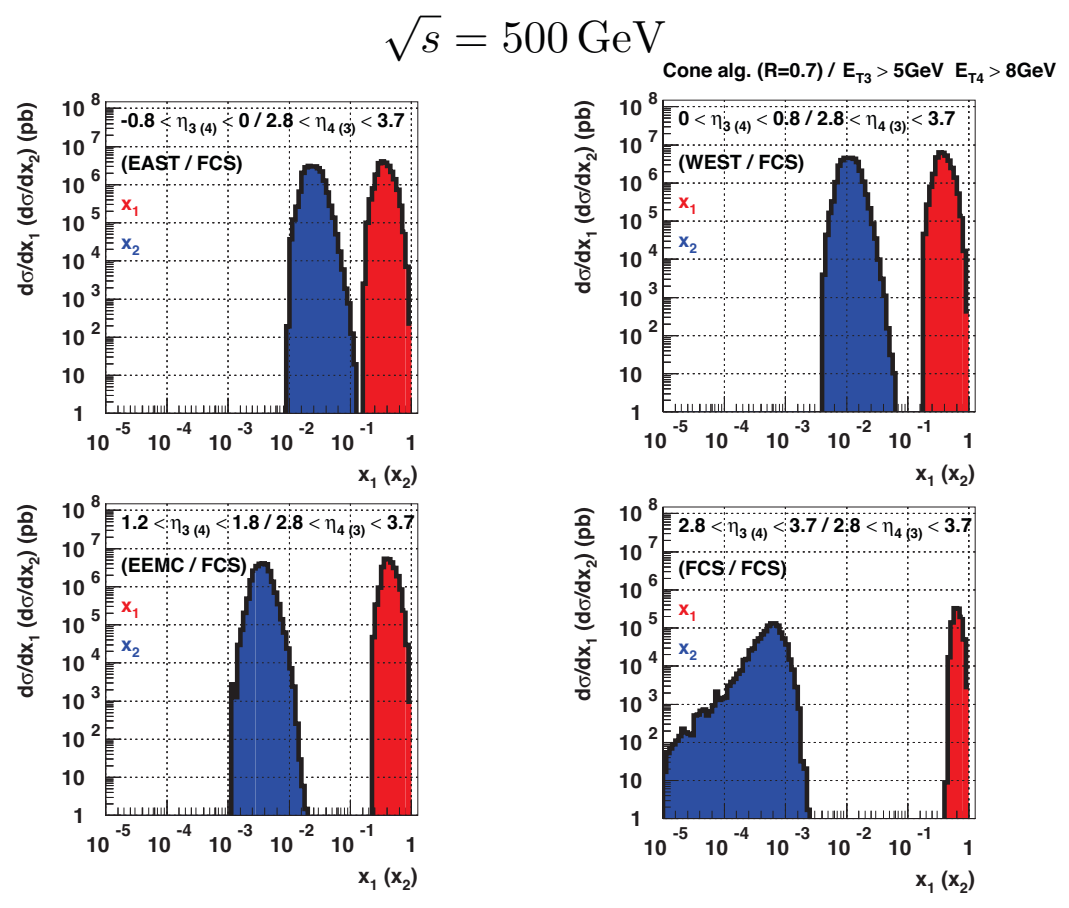

Figure 8. Parton momentum fractions, $x_{1}$ and $x_{2}$, probed by dijets with at least one jet in the forward STAR acceptance region in $\eta$ of $2.8<\eta<3.7$, with proposed FCS calorimeter upgrades.

Looking farther to the future, the STAR collaboration anticipates a fairly continuous evolution of the current detector into an eSTAR detector [36] for the future electron-ion collider (EIC) [37]. This machine will probe an enormous range of QCD and heavy-ion phenomena and will have spin physics capabilities, in polarized electron-proton collisions, with an unprecedented reach in $Q^{2}$ and $x$. One component of that future eSTAR detector, to be installed on an intermediate time frame (e.g. 2020) would be an upgraded Forward Calorimeter System (FCS) [38]. This system would involve a compact tungsten spaghetti calorimeter for an electromagnetic calorimeter and, for the first time at STAR, would involve significant hadronic calorimetry, with a lead-scintillating tile system, all covering $2.5<\eta<4.0$. This would allow forward jet measurements at STAR.

As one example of the proposed FCS functionality, we present projections for a dijet $A_{L L}$ measurement with $1 \mathrm{fb}^{-1}$ of data at $\sqrt{s}=500 \mathrm{GeV}$. Dijet and other two-to-two processes have the advantage that, at leading order, you can calculate the initial-state particle momenta. So, at leading order, the momentum fractions of the leading and sub-leading partons, $x_{1}$ and $x_{2}$, are identified. This proposed measurement, discussed in more detail elsewhere [38, 39], is summarized in Figs. 8 and 9. There, for various djiet topologies with at least one jet in the FCS acceptance, we present the parton $x$ probed along with the anticipated uncertainties on $A_{L L}$. The theoretically expected $A_{L L}$ values in this kinematic region are tiny, of order $10^{-3}$. But, the anticipated experimental uncertainties are equally minuscule, thus presenting an attractive possibility for probing the gluon polarization at extremely small $x$ before the EIC era. 


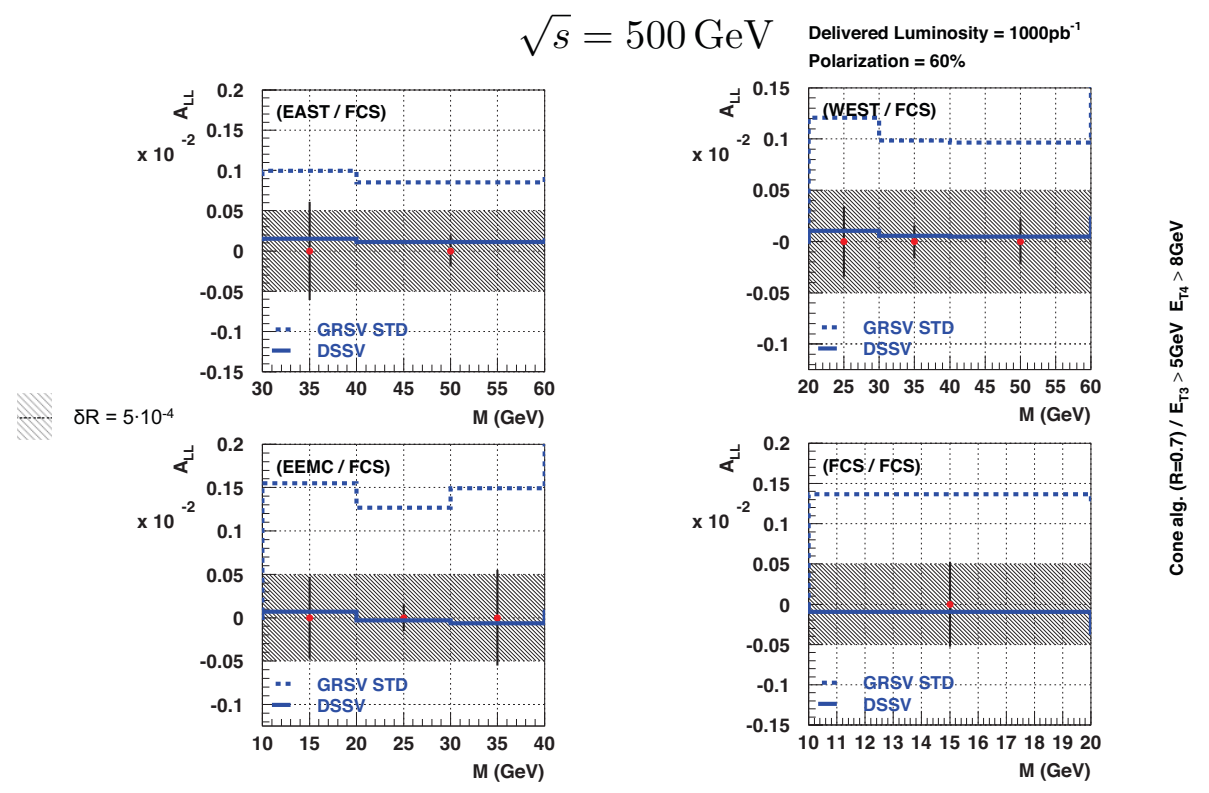

Figure 9. NLO $A_{L L}$ calculations for dijets with at least one jet having $2.8<\eta<3.7$, together with projected uncertainties.

\section{References}

[1] J. Ashman et al. (European Muon Collaboration), Phys.Lett. B206, 364 (1988)

[2] R. Klem, J. Bowers, H. Courant, H. Kagan, M. Marshak et al., Phys.Rev.Lett. 36, 929 (1976)

[3] C.A. Aidala, S.D. Bass, D. Hasch, G.K. Mallot, Rev.Mod.Phys. 85, 655 (2013), hep-ph/1209.2803

[4] K. Ackermann et al. (STAR Collaboration), Nucl. Inst. \& Meth. A499, 624 (2003), and references therein

[5] S. Shi (the STAR collaboration) (2014), in the Proceedings of the International Conference on New Frontiers in Physics 2014, nucl-ex/1409.8371

[6] D. de Florian, R. Sassot, M. Stratmann, W. Vogelsang, Phys.Rev.Lett. 101, 072001 (2008), hep-ph/0804.0422

[7] M. Cacciari, G.P. Salam, G. Soyez, J. High Energy Phys. 04, 063 (2008)

[8] L. Adamczyk et al. (STAR Collaboration) (2014), hep-ex/1405 . 5134

[9] D. de Florian, R. Sassot, M. Stratmann, W. Vogelsang, Phys.Rev.Lett. 113, 012001 (2014), hep-ph/1404.4293

[10] R. Sassot, M. Stratmann, private communication

[11] B. Abelev et al. (STAR Collaboration), Phys.Rev. D80, 111108 (2009), hep-ex/0911 . 2773

[12] S.W. Wissink (STAR Collaboration), AIP Conf.Proc. 1149, 397 (2009)

[13] L. Adamczyk et al. (STAR Collaboration), Phys.Rev. D89, 012001 (2014), nucl-ex/1309. 1800 
[14] E.R. Nocera, R.D. Ball, S. Forte, G. Ridolfi, J. Rojo (NNPDF Collaboration), Nucl.Phys. B887, 276 (2014), hep-ph/1406.5539

[15] C. Dilks (STAR Collaboration) (2014), presented at the 21st International Symposium on Spin Physics (Spin2014)

[16] M. Gluck, E. Reya, M. Stratmann, W. Vogelsang, Phys.Rev. D63, 094005 (2001), hep-ph/0011215

[17] L. Adamczyk et al. (STAR Collaboration), Phys.Rev.Lett. 113, 072301 (2014), nucl-ex/1404.6880

[18] E. Aschenauer, A. Bazilevsky, K. Boyle, K. Eyser, R. Fatemi et al. (2013), nucl-ex/1304 . 0079

[19] G.L. Kane, J. Pumplin, W. Repko, Phys.Rev.Lett. 41, 1689 (1978)

[20] A. Efremov, O. Teryaev, Yad. Fiz. 36, 242 (1982), [Sov. J. Nucl. Phys. 36, 140 (1982)]

[21] J.w. Qiu, G.F. Sterman, Phys.Rev. D59, 014004 (1998), hep-ph/9806356

[22] D. Sivers, Phys. Rev. D 41, 83 (1990)

[23] D. Sivers, Phys. Rev. D 43, 261 (1991)

[24] K. Kanazawa, Y. Koike, Phys.Lett. B720, 161 (2013), 1212 . 3071

[25] K. Kanazawa, Y. Koike, A. Metz, D. Pitonyak, Phys.Rev. D89, 111501 (2014), 1404.1033

[26] J.C. Collins, Nucl.Phys. B396, 161 (1993), hep-ph/9208213

[27] D. Boer, P. Mulders, F. Pijlman, Nucl.Phys. B667, 201 (2003), hep-ph/0303034

[28] R. Fatemi (STAR Collaboration), AIP Conf.Proc. 1441, 233 (2012), hep-ex/1206. 3861

[29] J.K. Adkins (STAR Collaboration) (2013), presented at the Annual Fall Meeting of the APS Division of Nuclear Physics

[30] J. Drachenberg (STAR Collaboration), EPJ Web Conf. 73, 02009 (2014)

[31] M. Anselmino, M. Boglione, U. D’Alesio, E. Leader, S. Melis et al., Phys.Rev. D73, 014020 (2006), hep-ph/0509035

[32] J.K. Adkins, J.L. Drachenberg (STAR Collaboration) (2014), presented at the 21st International Symposium on Spin Physics (Spin2014), proceedings forthcoming

[33] J.C. Collins, Phys.Lett. B536, 43 (2002), hep-ph/0204004

[34] S. Fazio, D. Smirnov (STAR), PoS DIS2014, 237 (2014)

[35] A. Vossen (STAR), AIP Conf.Proc. 1560, 519 (2013)

[36] L. Adamczyk et al. (STAR Collaboration) (2014), public STAR Note 592, https://drupal.star.bnl.gov/STAR/starnotes/public/sn0592

[37] A. Accardi, J. Albacete, M. Anselmino, N. Armesto, E. Aschenauer et al. (2012), 1212 . 1701

[38] L. Adamczyk et al. (STAR Collaboration) (2014), public STAR Note 605, https://drupal.star.bnl.gov/STAR/starnotes/public/sn0605

[39] B. Surrow (STAR Collaboration), PoS DIS2014, 241 (2014), hep-ex/1407 . 4176 